\title{
Efeitos de tecnologia no conhecimento, atitude e prática de gestantes para o parto
}

\author{
Effects of technology on knowledge, attitude and practice of pregnant women for childbirth
}

Ivna Silva Andrade ${ }^{1}$, Régia Christina Moura Barbosa Castro ${ }^{1}$, Karla de Abreu Peixoto Moreira ${ }^{1}$, Cristina Poliana Rolim Saraiva dos Santos ${ }^{1}$, Ana Fátima Carvalho Fernandes ${ }^{1}$

Objetivo: avaliar os efeitos de vídeo educativo sobre o conhecimento, a atitude e a prática de gestantes para o parto ativo. Métodos: estudo quase experimental, desenvolvido em dez unidades básicas de saúde da família, com 167 gestantes no último trimestre, por meio de instrumento construído e validado para levantamento do conhecimento, da atitude e prática. No Grupo Intervenção, a investigação foi aplicada, acompanhada de sessão educativa, com exposição de vídeo. Para o Grupo Controle, a investigação e a consulta pré-natal de rotina foram aplicadas. Resultados: o Grupo Intervenção apresentou maior adequação em conhecimento, atitude e prática, com estatísticas significativas em todas as variáveis, destacando a influência do vídeo educativo para o parto ativo. Conclusão: o uso de vídeo educativo se destacou positivamente como tecnologia educacional para conhecimento, atitude e prática de gestantes em relação ao parto ativo.

Descritores: Parto; Enfermagem Obstétrica; Tecnologia Educacional; Promoção da Saúde.

Objective: to evaluate the effects of educational video on the knowledge, attitude and practice of pregnant women for active childbirth. Methods: a quasi-experimental study, developed in ten basic family health units, with 167 pregnant women in the last trimester, through a built and validated instrument to survey knowledge, attitude and practice. In the Intervention Group, the research was applied, accompanied by an educational session, with video exposure. For the Control Group, routine prenatal investigation and consultation were applied. Results: the Intervention Group presented greater adequacy in knowledge, attitude and practice, with significant statistics in all variables, highlighting the influence of educational video for active childbirth. Conclusion: the use of educational video stood out positively as educational technology for knowledge, attitude and practice of pregnant women in relation to active childbirth.

Descriptors: Parturition; Obstetric Nursing; Educational Technology; Health Promotion.

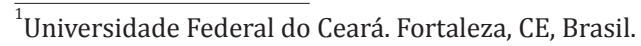




\section{Introdução}

O ciclo gravídico-puerperal ocasiona modificações em mulheres, logo a assistência à saúde deve primar pela qualidade de vida de gestantes, evitando futuras complicações futuras, por isso a importância da assistência pré-natal de alta qualidade, com acompanhamento de equipe multidisciplinar, assegurando, ao final da gestação, o nascimento de criança saudável e o bem-estar materno. O Ministério da Saúde do Brasil propõe política pública que contemple a melhoria do acesso, da cobertura e da qualidade do acompanhamento pré-natal, da assistência ao parto e ao binômio mãe e filho, com garantia dos direitos de cidadania, visando humanização de acolhimento digno à tríade mulher-bebê-família, a partir de condutas éticas e solidárias ${ }^{(1)}$.

Entretanto, na evolução da assistência à saúde, a tendência é o uso de tecnologias educacionais como ferramentas para ampliar o conhecimento e promover melhoramentos na qualidade de vida de usuários, uma vez que esses artifícios funcionam como dispositivos de mediação para os facilitadores, na disseminação da informação e formação, além de serem percebidos como forma de aproximação entre indivíduos, família, sociedade e profissionais da área, a partir de estratégias que garantem princípios do Sistema Único de Saúde ${ }^{(2-3)}$.

Classificadas em três categorias, as tecnologias podem ser duras, quando se utilizam de equipamentos tecnológicos, instrumentos, normas e rotinas; leve-dura, compreende todos os saberes bem estruturados no processo de saúde como modelos de cuidado, teorias; e leves, com uso de tecnologias de relações, produção, comunicação, acolhimento, vínculos e autonomização ${ }^{(4-5)}$.

Especificamente para mulheres no ciclo gravídico-puerperal, essas tecnologias contemplam a fisiologia do corpo feminino, práticas saudáveis na gestação, parto e puerpério, condutas adequadas e inadequadas nesse período, melhores formas de autocuidado, contemplando as necessidades destas. Para enfermeiros, as tecnologias educativas são necessárias no cotidiano da prática clínica, por ajudar no processo de comunicação e interação entre o profissional, a mulher e familiares, no estímulo, no esclarecimento e na orientação para impactar positivamente a vivência da maternidade ${ }^{(3-6)}$.

Mesmo com vasta literatura acerca do tema, o uso de tecnologias educativas para avaliar o conhecimento, a atitude e prática em relação ao parto ativo ainda é limitado, tornando o presente estudo relevante, uma vez que, ainda, coexistem muitas expectativas, dúvidas e incertezas quanto a esse tipo de parto. Assim, objetivou-se avaliar os efeitos de vídeo educativo no conhecimento, na atitude e prática de gestantes para o parto ativo.

\section{Métodos}

Estudo quase experimental, composto de grupo intervenção (GI) e grupo controle (GC) não equivalente, de desenho pré-teste/pós-teste, realizado em 10 Unidades Básicas de Saúde da Família, do município de Eusébio, Ceará, Brasil. A amostra proveniente desses dois territórios foi dividida em dois grupos: um território constituiu o grupo intervenção e o outro o grupo controle, por serem distantes entre si. A escolha das 10 unidades deu-se em virtude de os dois territórios englobarem o maior número de gestantes.

A população do estudo consistiu em 550 gestantes cadastradas nas referidas unidades que realizaram o exame de pré-natal, nos meses destinados para coleta de dados. Para o cálculo amostral, utilizou-se da fórmula para estudos de caso controle, desenhada para permitir determinar se uma exposição está associada com um desfecho ${ }^{(7)}$, considerando-se a razão de chance de 1,75 , a probabilidade de falta de experiência da prática em torno de $20,0 \%$, precisão relativa de 10,0\% e nível de significância de 5,0\%. Aplicando-se esses valores na fórmula, obteve-se $n=84$ para cada um dos grupos.

Para seleção das participantes, adotaram-se os seguintes critérios de inclusão: gestantes no terceiro 
trimestre, realizar consulta pré-natal nos dias marcados para coleta de dados e residir no município onde foi realizada a pesquisa. Os critérios de exclusão foram: encaminhamento para o parto cesáreo, já que o vídeo é voltado à preparação para o parto normal, tendo a necessidade de se avaliar a evolução e a finalização da parturiente para essa via de parto; e mães com deficiência auditiva e visual que a impedissem de participar da intervenção educativa e responder ao formulário avaliativo.

0 vídeo educativo aplicado tinha duração de 27 minutos, resultado de tese de Doutorado oriunda ao Programa de Pós-graduação da Universidade Federal do Ceará( ${ }^{(8)}$. A exposição desse material ocorreu por abordagem grupal, realizada pela pesquisadora em uma das Unidades Básica de Saúde, a qual disponibilizou recurso audiovisual, auditório silencioso, com ar condicionado e capacidade para acomodar confortavelmente 10 pessoas, para que pudessem ser captadas todas as informações repassadas. No total, foram realizados 15 grupos, até totalizar o quantitativo do cálculo amostral, ou seja, 84 gestantes em cada grupo.

Os conteúdos abordados no vídeo são: o conhecimento da gravidez mês a mês, os exercícios físicos com bola e cavalinho, respiração, massagem, presença de acompanhante, orientações dos profissionais no pré-natal e posicionamentos facilitadores do trabalho de parto e parto.

Para coleta de dados, foi construído e validado em conteúdo um inquérito sobre Conhecimento, Atitude e Prática (CAP) acerca da preparação para o parto ativo e realização deste, com quesitos avaliativos de respostas variando de adequado a inadequado, no total de sete questões, mediante revisão e análise do estado da arte acerca da temática. Este foi validado por três juízes, enfermeiros, doutores, com pesquisas publicadas na área, envolvidos na assistência em relação à saúde materna e docentes de um curso de graduação em Enfermagem. $\mathrm{O}$ instrumento foi analisado quanto à forma de apresentação e ao conteúdo elaborado. As sugestões enviadas foram lidas e as alterações nas questões foram realizadas, bem como nas categorias das respostas e quanto à retirada e/ou acréscimo de algumas questões.

$\mathrm{Na}$ categoria sobre conhecimento, a pergunta norteadora foi: o que caracteriza um parto ativo? A indagação: o que você acha importante fazer para ter um parto ativo? respondeu à questão para desvelar a atitude.

Com o recrutamento das gestantes, foi realizado o convite para participação no estudo. Ao aceite e assinatura do Termo de Consentimento Livre Esclarecido, os grupos foram formados. No GI, composto por 84 gestantes, realizou-se consulta pré-natal de rotina, com posterior exposição do vídeo educativo e entrevista com formulário sobre CAP acerca da preparação para o parto ativo e realização deste; para o GC, com 83 gestantes, foram utilizados os mesmos procedimentos do GI, com exceção da exposição do vídeo educativo, totalizando 10 encontros.

O banco de dados foi desenvolvido no programa estatístico Statistical Package for the Social Sciences, versão, 20.0. A análise exploratória dos dados constou de testes estatísticos descritivos, frequência absoluta e relativa, apresentados por meio de tabelas. Para análise da correlação das variáveis sociodemográficas e obstétricas, com o conhecimento, a atitude e prática, utilizou-se do teste de Fisher. Quanto à avaliação das categorias de respostas (conhecimento, atitude e prática) e do grupo estudado, utilizou-se do teste Qui-Quadrado.

0 estudo foi conduzido de acordo com as normas da Resolução 466/12 sobre pesquisas envolvendo seres humanos e aprovado pelo Comitê de Ética em Pesquisa da Universidade Federal do Ceará, conforme parecer no 1.148.019.

\section{Resultados}

As participantes do estudo totalizaram 167 gestantes, distribuídas entre GI (84 gestantes) e GC (83 gestantes), na qual a maioria tinha entre 20 e 30 anos (59,8\%), viviam com companheiro (86,8\%), o tempo de estudo foi menor que 12 anos $(65,2 \%)$, primíparas 
(48,5\%), com início do pré-natal ainda no primeiro trimestre $(92,2 \%)$.

Quanto à avaliação da adequação do conhecimento, da atitude e prática dos grupos, percebe-se que o GI apresentou maior adequação dos quesitos, com significância estatística em quase todas as variáveis, totalizando resultado de 50, 62 e 71,4\%, respectivamente, quando associadas ao grupo que recebeu a tecnologia ( $\mathrm{p}=0,001, \mathrm{p}=0,044 \mathrm{e} \mathrm{p}=0,054)$.

A Tabela 1 retrata a avaliação do nível de conhecimento dos grupos em questão, quando indagadas: sobre o que caracteriza um parto ativo. Observou-se que, comparando as respostas do GC e do GI, obteve-se que a adequação foi maior no segundo grupo, cuja tecnologia por vídeo foi aplicada. As respostas mais citadas pelas gestantes do GI foram a participação ativa da mãe $(57,1 \%)$ e do acompanhante de livre escolha (42,9\%). Opondo-se às respostas consideradas inadequadas, as mulheres do GC demonstraram desconhecimento quanto à posição vertical $(95,2 \%)$ e privacidade na hora de parir $(89,2 \%)$ como pontos importantes para o parto ativo.

Tabela 1 - Avaliação dos grupos quanto ao nível de conhecimento acerca do parto ativo

\begin{tabular}{lcccc}
\hline \multirow{2}{*}{$\begin{array}{l}\text { O que caracteriza um parto } \\
\text { ativo? }\end{array}$} & \multicolumn{2}{c}{ Controle } & \multicolumn{2}{c}{ Intervenção } \\
\cline { 2 - 5 } & $\begin{array}{l}\text { Adequa- } \\
\text { do n (\%) }\end{array}$ & $\begin{array}{c}\text { Inadequa- } \\
\text { do n (\%) }\end{array}$ & $\begin{array}{c}\text { Adequa- } \\
\text { do n (\%) }\end{array}$ & $\begin{array}{c}\text { Inadequa- } \\
\text { do (\%) }\end{array}$ \\
\hline Participação ativa da mãe & 34,9 & 65,1 & 57,1 & 42,9 \\
Participante do acompanhante & 14,5 & 85,5 & 42,9 & 57,1 \\
Uso da posição vertical & 4,8 & 95,2 & 11,9 & 88,1 \\
Privacidade na hora de parir & 10,8 & 89,2 & 26,2 & 73,8 \\
Uso de terapias não farmacoló- & 15,7 & 84,3 & 13,1 & 86,9 \\
gicas para o parto & & & & \\
Não sabe ou não lembra & 30,1 & 69,9 & 25,0 & 75,0 \\
\hline
\end{tabular}

Na Tabela 2, quando questionadas sobre o que considerava importante fazer para ter um parto ativo, o item mais respondido pelas gestantes do GI foi respiração $(76,2 \%)$, seguida do uso da bola e do cavalinho $(45,2 \%)$. Merece atenção o desconhecimento das mulheres do GC, pois a maioria não assinalou práticas importantes no momento do parto, como deambulação $(84,3 \%)$ e posição vertical $(89,2 \%)$.
Tabela 2 - Avaliação dos grupos quanto ao nível de atitude acerca do parto ativo

\begin{tabular}{|c|c|c|c|c|}
\hline \multirow{2}{*}{$\begin{array}{l}\text { O que você acha im- } \\
\text { portante fazer para } \\
\text { ter um parto ativo? }\end{array}$} & \multicolumn{2}{|c|}{ Controle } & \multicolumn{2}{|c|}{ Intervenção } \\
\hline & $\begin{array}{c}\text { dequad } \\
\text { n (\%) }\end{array}$ & $\begin{array}{l}\text { Inadequa- } \\
\text { do } n(\%)\end{array}$ & $\begin{array}{c}\text { Adequado } \\
\text { n (\%) }\end{array}$ & $\begin{array}{r}\text { Inadequa } \\
\text { do } n(\%)\end{array}$ \\
\hline Respiração & 60,2 & 39,8 & 76,2 & 23,8 \\
\hline Deambulação & 15,7 & 84,3 & 28,6 & 71,4 \\
\hline Posição vertical & 10,8 & 89,2 & 20,2 & 79,8 \\
\hline Uso de bola e cavalinho & 34,9 & 65,1 & 45,2 & 54,8 \\
\hline Não sabe ou não lembra & 20,5 & 79,5 & 10,7 & 89,3 \\
\hline
\end{tabular}

Na Tabela 3, observou-se significância estatística nas variáveis participação ativa da mãe $(\mathrm{p}=0,005)$, participação do acompanhante de livre escolha $(\mathrm{p}=0,000)$ e privacidade na hora de parir $(\mathrm{p}=0,016)$, que corresponderam às variáveis relacionadas ao nível de conhecimento acerca do parto ativo. Com relação ao nível atitude, houve diferença entre os grupos no item respiração $(\mathrm{p}=0,031)$. As variáveis relacionadas à prática ao parto ativo não apresentaram significância estatística.

Tabela 3 - Avaliação das categorias questionadas quanto ao conhecimento, à atitude e prática nos dois grupos

\begin{tabular}{lc}
\hline Pergunta & $\mathbf{p}^{*}$ \\
\hline O que caracteriza um parto ativo? & 0,005 \\
Participação ativa da mãe & 0,000 \\
Participação do acompanhante de livre escolha & 0,161 \\
Uso da posição vertical & 0,016 \\
Privacidade na hora de parir & 0,665 \\
Uso de terapias não farmacológicas para o parto & \\
Se seu parto foi normal, quais foram suas atitudes & 0,159 \\
Respiração & 0,565 \\
Deambulação & 1,000 \\
Posição vertical & 1,000 \\
Uso da bola e cavalinho & 1,000 \\
Não sabe ou não tem opinião & \\
Tipo de parto & 0,861 \\
Qual o tipo de parto pretendido (Atitude) & 0,497 \\
Qual o tipo de parto teve (Prática) &
\end{tabular}




\section{Discussão}

Com implicações para prática de enfermagem e saúde, os resultados apontaram que o uso de tecnologia educativa por meio de vídeo contribui para o desenvolvimento da atitude crítico-reflexiva de gestantes, além de colaborar para o conhecimento acerca do processo parturitivo.

Os achados encontrados neste estudo são apoiados por outros estudos que também mostram resultados promissores quando se trata do acesso às informações para conhecimento sobre a melhor via de parto, medidas não farmacológicas durante o parto, melhores condições de nascimento para o bebê, recuperação no pós-parto ${ }^{(9-11)}$.

Pesquisadores de maternidade de Minas Gerais, Brasil, evidenciaram que entre 40 parturientes, os cuidados mais registrados na assistência ao parto foram o exercício respiratório, banho de aspersão, deambulação, mudanças de posições (cócoras, sentada, agachamento), massagens e bola suíça ${ }^{(12)}$, resultados semelhantes encontrados no estudo.

Diante desses cuidados, as características das práticas de cuidado interferem positivamente na qualidade da assistência à saúde, já que percebem esse evento como processo fisiológico natural. 0 entendimento que a humanização e a qualidade na assistência à mulher são ampliadas a partir de adoção de métodos não farmacológicos para alívio da dor, reforça-se, segundo estudo com 119 puérperas de maternidade pública, referência na assistência materna e infantil no Estado do Ceará(11,13).

Cada vez mais as práticas e os serviços de atenção ao parto são influenciados pelos papéis desempenhados pela parturiente, pelos profissionais que a assistem e pelo ambiente onde ocorre o evento. Locais com atendimento voltado à fisiologia do nascimento e parto, cujas enfermeiras obstétricas e obstetrizes têm autonomia para desenvolver habilidades para promover o parto normal, permitem o uso de boas práticas, proporcionando conforto, tranquilidade, menos an- siedade e redução de práticas abusivas ${ }^{(14)}$.

Nesse contexto, em estudo realizado em maternidade de médio porte no estado do Rio Grande do Sul, Brasil, observou-se que as gestantes dão importância ao conhecimento adquirido por meio de experiências pessoais anteriores, relatos e vivências de familiares e saberes compartilhados ao longo das trajetórias de vida, quando falavam da forma como foi despertado o desejo pelo tipo de parto atual ${ }^{(11)}$.

No entanto, muitas vezes, quando o atendimento humanizado não é concretizado, como citado em estudo realizado em sete maternidades públicas do Centro-Oeste de Minas Gerais, sinaliza-se que a opção pela via de parto não foi por escolha da parturiente, e sim pela determinação do profissional de saúde, descaracterizando o atendimento humanizado nesse momento ${ }^{(10)}$.

Nessa perspectiva, a prática de tecnologias adequadas na assistência ao parto e nascimento, a partir de modelo de assistência humanizada, com ênfase em evidências científicas, resgatando o empoderamento de mulheres com o emprego de práticas não medicamentosas de alívio à dor do parto e respeitando à fisiologia do parto, causam menos danos para mãe e bebê e podem permitir à mulher mais sensação de autonomia no parto ${ }^{(4,15-16)}$.

No Brasil, o Ministério da Saúde destaca as Políticas Públicas em Atenção à Saúde da Mulher, regulamentando o direto ao planejamento reprodutivo e a atenção humanizada à gravidez ao parto e ao puerpério (pós-parto), bem como direito ao nascimento seguro e ao crescimento e desenvolvimento saudáveis. Propostas que buscam a qualificação dos serviços oferecidos pelo Sistema Único de Saúde desde o planejamento familiar, na confirmação da gravidez, no pré-natal, parto e puerpério, considerando 28 dias após o parto ${ }^{(17) .}$

Ao entender que a assistência pré-natal objetiva reduzir a morbimortalidade materno-fetal, os cuidados deverão ser recebidos desde o período gestacional até o minuto do parto. Além disso, o pré-natal 
não se baseia apenas em consultas e solicitação de exames, inclui, também, a realização de estratégias que envolvam o acolhimento e o reconhecimento das necessidades de saúde que contribuam para o estabelecimento de vínculos da gestante com a equipe de saúde ${ }^{(18)}$.

Assim, surge o plano de parto como mais um aliado à participação ativa da gestante durante o processo de parturição. Traça-se, portanto, plano para o casal, instrumento educativo que informa sobre todas as alternativas disponíveis na assistência ao parto, com ou sem irregularidades. 0 plano poderá ser mudado de acordo com as práticas dos serviços, o que possibilitará à mulher ter autonomia na escolha da via de parto e, ao mesmo tempo, ser protagonista da própria história ${ }^{(11)}$.

\section{Conclusão}

Os achados deste estudo apontaram que a tecnologia educativa do tipo vídeo obteve efeito exitoso no conhecimento, na atitude e prática de gestantes acerca do parto ativo, destacando-se como aliado nas implicações para prática de enfermagem e saúde.

\section{Agradecimentos}

Ao Conselho Nacional de Desenvolvimento Científico e Tecnológico, pela concessão de bolsa de Produtividade em Pesquisa a Ana Fátima Carvalho Fernandes, processo no 301943/2017-6.

\section{Colaborações}

Andrade IS contribuiu com concepção do projeto, análise e interpretação dos dados e redação do artigo. Castro RCMB e Moreira KAP colaboraram com revisão crítica relevante do conteúdo intelectual. Santos CPRS e Fernandes AFC contribuíram com redação do artigo e aprovação final da versão final a ser publicada.

\section{Referências}

1. Possati AB, Prates LA, Cremonese L, Scarton J, Alves CN, Resse LB. Humanization of childbirth: meanings and perceptions of nurses. Esc Anna Nery. 2017; 21(4):e20160366. doi: http://dx.doi. org/10.1590/2177-9465-ean-2016-0366

2. Mangini FNR, Kocourek S, Silveira LV. Serviço social e tecnologias de saúde: o desafio da subversão democrática. Serv Soc Saúde. 2018; 17(1):65-94. doi: https://doi.org/10.20396/sss. v17i1.8655203

3. Quental LLC, Nascimento LCCC, Leal LC, Davim RM, Cunha ICBC. Educational practices with pregnant women at a primary health care. Rev Enferm UFPE on line. 2017; 11(Supl. 12):537081. doi: https://doi.org/10.5205/1981-8963v11i12a23138p5370-5381-2017

4. Duarte MR, Alves VH, Rodrigues DP, Souza KV, Pereira AV, Pimentel MM. Care technologies in obstetric nursing: contribution for the delivery and birth. Cogitare Enferm. 2019; 24:e54164. doi: http://dx.doi.org/10.5380/ce.v24i0.54164

5. Sabino LMM, Brasil DRM, Caetano JA, Santos MCL, Alves MDS. Uso de tecnologia leve-dura nas práticas de enfermagem: análise de conceito. Aquichan. 2016; 16(2):230-9. doi: http://dx.doi. org/10.5294/aqui.2016.16.2.10

6. Barbosa EMG, Sousa AAS, Vasconcelos MGF, Carvalho REFL, Oriá MOB, Rodrigues DP. Educational technologies to encourage (self) care in postpartum women. Rev Bras Enferm. 2016; 69(3):54553. doi: http://dx.doi.org/10.1590/0034$7167.2016690323 \mathrm{i}$

7. Polit DF, Beck CT. Fundamentos de pesquisa em enfermagem: avaliação de evidências para a prática da enfermagem. Porto Alegre: Artmed; 2018.

8. Moreira KAP.Tecnologia educativapara a promoção do conforto, privacidade e autonomia da gestante para um parto ativo [Internet]. 2014 [citado 2019 ju 13]. Disponível em: http://www.repositorio. ufc.br/bitstream/riufc/21935/1/2016_tese_ isandrade.pdf

9. Silva AS, Oliveira CCC, Brito ECC, Castro e Silva TB, Evangelista DR. Conhecimento das gestantes acerca das medidas de alívio da dor durante o parto. 
Rev Enferm Atual [Internet]. 2019 [citado 2019 Jun 5];87(Supp):1-7. Disponível em: https://revistaenfermagematual.com.br/index.php/revista/article/view/221/122

10. Oliveira VJ, Penna CMM. Every birth is a story: process of choosing the route of delivery. Rev Bras Enferm. 2018; 71(suppl 3):1304-12. doi: http:// dx.doi.org/10.1590/0034-7167-2016-0497

11. Prates LA, Timm MS, Wilhelm LA, Cremonese GO, Schimith MD, Ressel LB. Being born at home is natural: care rituals for home birth. Rev Bras Enferm. 2018; 71(suppl 3):1324-34 doi: http:// dx.doi.org/10.1590/0034-7167-2017-0541

12. Dias EG, Ferreira ARM, Martins AMC, Jesus MM, Alves JCS. Eficiência de métodos não farmacológicos para alívio da dor no trabalho de parto normal. Enferm Foco [Internet]. 2018 [citado 2019 Jun 5];9(2):35-39. Disponível em: http://revista. cofen.gov.br/index.php/enfermagem/article/ view/1398/442

13. Melo LPT, Pereira AMM, Rodrigues DP, Dantas SLC, Ferreira ALA, Fontenele FMC, et al. Representações de puérperas sobre o cuidado recebido no trabalho de parto e parto. Av Enferm. 2018; 36(1):2230. doi: http://dx.doi.org/10.15446/av.enferm. v36n1.63993

14. Gonçalves LD, Silva JC, Rodrigues MS. Boas práticas na assistência ao parto: implicações do plano de parto. Rev Bras Ciênc Vida [Internet]. 2018 [citado 2019 Jun 5];6(n. esp):1-27. Disponível em: http:// jornal.faculdadecienciasdavida.com.br/index. php/RBCV/article/view/747/32

15. Souza RG, Silva AK, Rodrigues JMS, Kawakame PMG. Educação em saúde sobre o uso de tecnologias não invasivas para alívio da dor no trabalho de parto. PECIBES [Internet]. 2018 [citado 2019 Jun 5];4(2):57-101. Disponível em: http:// www.seer.ufms.br/index.php/pecibes/article/ view/6946/5104

16. Guida NFB, Pereira ALF, Lima GPV, Zveiter M, Araújo CLF, Moura MAV. Compliance of nursing care practices with technical recommendations for normal birth. Rev Rene. 2017; 18(4):54350. doi: http://dx.doi.org/10.15253/21756783.2017000400017

17. Ministério da Saúde (BR). Rede cegonha [Internet]. 2017 [citado 2019 Jun 5]. Disponível em: http:// portalms.saude.gov.br/saude-para-voce/saudeda-mulher/rede-cegonha

18. Mayor MSS, Herrera SDSC, Araujo MQA, Santos FM, Arantes RV, et al. Avaliação dos Indicadores da Assistência Pré-Natal em Unidade de Saúde da Família, em um município da Amazônia Legal. Rev Cereus [Internet]. 2018 [citado 2019 Jun 5];10(1):91-100. Disponível em: http://ojs.unirg. edu.br/index.php/1/article/view/2079/636 